\title{
Interleukin-2 augmented activation of tumor associated macrophage plays the main role in MHC class I in vivo induction in tumor cells that are MHC negative in vitro
}

\author{
GUAN-FENG OUYANG ${ }^{1}$, MASANAO SAIO ${ }^{1}$, TATSUHIKO SUWA ${ }^{1,2}$, HISASHI IMAI ${ }^{1,3}$, JIRO NAKAGAWA ${ }^{1,4}$, \\ KENICHI NONAKA ${ }^{1,3}$, NAOKI UMEMURA ${ }^{1,2}$, MIKA KIJIMA $^{5}$ and TSUYOSHI TAKAMI ${ }^{1}$ \\ Departments of ${ }^{1}$ Immunopathology, ${ }^{2}$ Oral and Maxillofacial Sciences, ${ }^{3}$ Oncologic Surgery and ${ }^{4}$ Neurosurgery, \\ Gifu University Graduate School of Medicine, Gifu; ${ }^{5}$ Department of Immunology and Parasitology, \\ Institute of Health Biosciences, The University of Tokushima Graduate School, Tokushima, Japan
}

Received November 28, 2005; Accepted January 9, 2006

\begin{abstract}
The contribution of tumor associated macrophage (TAM) to the induction of major histocompatibility complex (MHC) class I expression in vivo has not been reported precisely. In this study, we utilized Interleukin-2 (IL-2) cDNAintroduced B16 melanoma cells (B16/IL-2) and vehicle-alone control cells (B16/mock) to examine whether TAM could contribute to the induction of MHC class I on B16 cells in vivo. Interestingly, although B16/mock and B16/IL-2 did not express MHC class I in vitro, MHC class I was strongly expressed in vivo in B16/IL-2 in comparison to B16/mock. Although in vivo treatment of anti-NK1.1 antibody abolished MHC expression in B16/mock in vivo, the same treatment did not influence MHC expression in B16/IL-2. Interestingly, both anti-asialo GM1 and anti-CD11b treatment strongly decreased MHC expression in B16/IL-2. TAM expressed both asialo GM1 and CD11b antigen, and TAM recovered from B16/IL-2 produced interferon $\gamma$ (IFN $\gamma) 6$ times more than that from B16/mock. In addition, TAM recovered from B16/IL-2 secreted 33.64 times more IFN $\gamma$ in response to in vitro administration of IL-2. Therefore, we checked whether or not IL-2 could influence the expression of IL-2 receptors. TAM recovered from IL-2 expressed middle affinity receptor of IL-2 (CD122 and CD132) while that from B16/mock expressed low affinity receptor (CD25 and CD132). Finally, we observed that $\mathrm{B} 16$ cells became apoptotic with IFN $\gamma$ treatment in vitro. These results suggested that IL-2 augmented activation of TAM would play the main role in induction of the MHC class I
\end{abstract}

Correspondence to: Dr Masanao Saio, Department of Immunopathology, Gifu University Graduate School of Medicine, 1-1 Yanagido, Gifu, Gifu 501-1194, Japan

E-mail: saio@cc.gifu-u.ac.jp

Key words: tumor associated macrophage, Interleukin-2, mouse, major histocompatibility complex molecule through secretion of IFN $\gamma$, and would contribute to the IFN $\gamma$-mediated apoptosis induction in tumor cells.

\section{Introduction}

In the tumor environment, tumor associated macrophage (TAM) is the largest population in number of immune cells (1) and is alternatively activated, causing tumor immunesuppression (2). For example, Interleukin-10 (IL-10) produced in TAM suppresses production of IL-12 (3). Therefore, many researchers have tried to make TAM change from pro-tumor phenotype to anti-tumor phenotype using various strategies. For example, recombinant IL-2 was utilized to enhance macrophage mediated cytotoxicity against tumor cells $(4,5)$.

IL-2 is described as a factor that induces selective T-cell growth and maintains the survival of human $\mathrm{T}$ cells (6). Human IL-2 was cloned in 1983 by Taniguchi et al (7) and is extensively utilized for tumor treatment (8). The mechanisms exploited by IL-2 immunotherapy are categorized as direct effect and indirect effect. Induction of proliferation and stimulation of natural killer (NK) cells, lymphokine activated killer (LAK) cells, and tumor specific T cells are categorized as direct effects (9-12). Upregulation of major histocompatibility complex (MHC) molecules and adhesion molecules on endothelial cells, T cells, tumor cells and antigen presenting cells (APC) is categorized as an indirect effect (11).

In order to further explore how IL-2 affects the anti-tumor immune mechanism, we prepared IL-2 cDNA introduced B16 murine melanoma cells. We previously reported that NK cells played a critical role in inducing MHC class I expression on tumor cells in vivo (13). In the present study, we focused on the effect of IL-2 on TAM, especially whether or not TAM could affect the induction of MHC class I molecule expression on tumor cells. We also tried to confirm how TAM contributed to tumor immunity.

\section{Materials and methods}

Mice. Male C57BL/6 (B6) mice, 4-8 weeks old, were purchased from Charles River Japan (Kanagawa, Japan). The mice were 
maintained in accordance with the guidelines of the Committee on Animals of Gifu University School of Medicine.

Cell lines. Mouse melanoma cell line, B16, was purchased from Health Science Research Resources Bank (HSRRB; Osaka, Japan). Hybridoma cell lines (2.4G2: anti-mouse CD16/32, M1/70: anti-CD11b, PK136: anti-NK1.1) were obtained from American Type Culture Collection (Manassas, VA). Cells were cultured in RPMI-1640 medium containing $10 \%$ fetal calf serum (FCS), L-glutamate (Gibco-BRL, Rockville, MD), and penicillin-streptomycin (Gibco-BRL). The cells were maintained at $37^{\circ} \mathrm{C}$ in a humidified $5 \% \mathrm{CO}_{2}$ atmosphere. Murine IL-2 cDNA or vector vehicle alone was transferred into the B16-wild-type (B16-wt) as described below.

cDNA preparation and plasmid construction. Native spleen cells were prepared from normal B6 mice, and stimulated with $1 \mathrm{nM}$ phorbol miristate 13 acetate (Sigma, St. Louis, $\mathrm{MO}$ ) and $1 \mathrm{nM}$ calcium ionophore (Sigma) in complete RPMI-1640 medium (Gibco-BRL) for $12 \mathrm{~h}$. Total RNA of the splenocytes was prepared by Isogen (Nippon Gene, Tokyo, Japan) and then reverse-transcribed by M-MLV reversetranscriptase (Gibco-BRL). cDNA was amplified with an expanded long template polymerase chain reaction (PCR) system (Roche Diagnostics, Mannheim, Germany). The primer pair used to amplify the open reading frames of murine IL-2 was as follows: upper primer, 5'-CAC CCT TGC TAA TCA CTC CTC AC-3'; lower primer, 5'-GCG CTT ACT TTG TGC TGT CCT A-3'.

Transfection. The amplified cDNA was cloned into a pCR2.1 cloning vector (Invitrogen, Carlsbad, CA). JM109 E. coli (Takara, Seta, Japan) was transformed with the vector. Plasmid DNA was then purified with Qiagen-tip 500 (Qiagen, Hilden, Germany). The plasmid DNA containing an open reading frame of IL-2 and pcDNA3.1 hyg (Invitrogen) was digested at the BstXI site and the digested plasmid was electrophoresed on $1 \%$ agarose gel for $30 \mathrm{~min}$ at $100 \mathrm{mV}$. After staining with $0.1 \mu \mathrm{g} / \mathrm{ml}$ of ethidium bromide (Sigma), inserted cDNA fragments or linearized plasmids were sliced and collected, and the DNA was purified with a Gene Clean II kit (New England BioLabs, Boston, MA). Linearized pcDNA3.1 fragment was then dephosphorylated by thermosensitive alkaline phosphatase (Gibco) for $15 \mathrm{~min}$ at $65^{\circ} \mathrm{C}$ followed by another 15 -min stopping step at $65^{\circ} \mathrm{C}$. Two hundred nanograms of cDNA fragment and $100 \mathrm{ng}$ of pcDNA3.1 were ligated by $10 \mathrm{U} / \mathrm{ml}$ of T4 DNA ligase (Takara) for $16 \mathrm{~h}$ at $16^{\circ} \mathrm{C}$. After transformation into JM109, the proper clone was picked and checked by restriction enzyme analyses. Plasmid DNA was then purified with Qiagen-tip 500 (Qiagen). Purified plasmid was introduced into B16-wt cells using Lipofectamine plus reagent (Invitrogen) following the manufacturer's instructions manual. Transformed cells were selected by a culture medium containing $0.2 \mathrm{mg} / \mathrm{ml}$ of hygromycin B (Gibco), and then a limiting dilution method was performed to establish a single cell derived cell line. Finally, we obtained the IL-2 secreting clone, B16/IL-2\#28 (B16/IL-2), and mock transfectant, B16/mock. The cell lines used in this study showed the same growth speed in vitro (data not shown).
Enzyme linked immunosorbent assay (ELISA). ELISA was performed to determine the concentrations of IL-2 and interferon gamma $(\mathrm{IFN} \gamma)$ in the supernatants. ELISA assay plates (Nunc-Maxisorb $^{\circledR}$, Japan InterMed, Tokyo, Japan) were coated with $50 \mu 1$ of $2 \mu \mathrm{g} / \mathrm{ml}$ capture antibody in $0.1 \mathrm{M}$ carbonate buffer, $\mathrm{pH} 8.4$ for $16 \mathrm{~h}$ at $4^{\circ} \mathrm{C}$, and then the remaining active sites were blocked with $300 \mu 1$ of $10 \%$ FCS containing PBS, $\mathrm{pH} 7.0$ (dilution buffer) for $2 \mathrm{~h}$ at room temperature. Culture supernatant $(100 \mu \mathrm{l})$ was applied and incubated for $16 \mathrm{~h}$ at $4^{\circ} \mathrm{C}$ and then $100 \mu \mathrm{l}$ of $1 \mu \mathrm{g} / \mathrm{ml}$ biotinylated detection antibody was applied and incubated for $1 \mathrm{~h}$ at room temperature. One hundred microlitres of $2.5 \mu \mathrm{g} / \mathrm{ml}$ avidin-conjugated peroxidase (A-3151, Sigma) was applied and incubated for $30 \mathrm{~min}$ at room temperature and then the reaction was visualized with $100 \mu 1$ of TMB one step substrate system (Dako Japan, Kyoto, Japan). The reaction was terminated with $100 \mu \mathrm{l}$ of $3 \mathrm{~N} \mathrm{H}_{2} \mathrm{SO}_{4}$ and absorbance was measured at $450 \mathrm{~nm}$ with a $655-\mathrm{nm}$ reference by Benchmark microplate reader (Bio-Rad) and then analyzed using MPM-III software (Bio-Rad). At each step, the plate was washed with $0.05 \%$ Tween-20 in PBS, pH 7.0 by a model 1575 ImmunoWash $^{\circledR}$ microplate washer (Bio-Rad). JES6-1A12 rat anti-mouse IL-2 antibody or R4-6A2 rat anti-mouse IFN $\gamma$ antibody (Pharmingen, San Diego, CA) was used for capture and biotin-conjugated JES6-5H4 rat anti-mouse IL-2 antibody or XMG1.2 rat anti-mouse IFN $\gamma$ antibody was used for detection (Pharmingen). As a standard, we used recombinant mouse IL-2 or IFN $\gamma$ (Pharmingen). For the measurement of IL-2, 24-h cultured supernatants containing $5 \times 10^{5}$ cells $/ \mathrm{ml}$ of B16/mock and B16/IL-2 were measured. For the measurement of IFN $\gamma, 24-\mathrm{h}$ cultured supernatants containing $3 \times 10^{6}$ cells $/ \mathrm{ml}$ of tumor associated macrophages were measured.

Subcutaneous inoculation of tumor cells. Cells were harvested from the culture flask, washed three times with PBS and resuspended at $1 \times 10^{7}$ cells $/ \mathrm{ml}$ in PBS. One million cells were inoculated subcutaneously into the right flank of each mouse.

Tumor associated macrophage and tumor cell rich fraction preparation. Mice were sacrificed 10 days after each tumor implantation for analysis. Cell preparation was performed as described previously (14). In brief, 10 days after each tumor implantation, the tumor was collected and minced into small pieces before incubation with a mixture of enzymes dissolved in HBSS [collagenase Type I $(0.05 \mathrm{mg} / \mathrm{ml})$, collagenase Type IV $(0.05 \mathrm{mg} / \mathrm{ml})$, hyaluronidase $(0.025 \mathrm{mg} / \mathrm{ml})$ - all from Sigma, and DNase I $(0.01 \mathrm{mg} / \mathrm{ml})$ and soybean trypsin inhibitor (0.2 TIU/ml) - both from Roche Diagnostics] for $15 \mathrm{~min}$ at $37^{\circ} \mathrm{C}$ twice. Digested cells were harvested and red blood cells were lysed with red blood cell lysis buffer for $1 \mathrm{~min} . \mathrm{CD}_{11} \mathrm{~b}^{+}$ cells were separated using type $\mathrm{MS}^{+}$or $\mathrm{LS}^{+}$columns and anti-CD11b conjugated magnetic beads, according to the manufacturer's instructions (MACS, Miltenyi Biotec, BerdishGladbach, Germany). In brief, $10 \mu 1$ of beads suspension was added to $10^{7}$ cells in a final volume of $0.1 \mathrm{ml}$ cold HBSS (containing $0.5 \% \mathrm{FCS}$ ). After incubation for $15 \mathrm{~min}$ in the refrigerator, cells were washed and passed through a separation column. The column-binding population was collected as the $\mathrm{CD}_{1} 1 \mathrm{~b}^{+}$macrophage enriched fraction. 
To enrich tumor cells from total tumor digest, anti-CD11b, anti-DX5, anti-CD8a, anti-CD4, and anti-B220 magnetic beads were utilized to deplete immune cells from the digest, and the negative population was utilized for further analyses. We further clarified that over $90 \%$ of the cells in the population expressed melanoma antigen MM2-9B6 (data not shown) (13).

Preparation and labeling of antibody. Crude immunoglobulin fraction of anti-asialo-GM1 serum was purchased from Wako (Osaka, Japan) and then purified with Protein $G$ affinity column. After dialyzing to PBS, the protein concentration was determined and the fraction was labeled with a phycoerythrin (PE)-labeling kit (Dojindo, Kumamoto, Japan).

Flow cytometric analysis. All antibodies, except rat antimurine mature macrophage antibody (F4/80, rat IgG2b, Caltag, San Diego, CA) and rat anti-macrophage antibody (ER-MP20, rat IgG2a, BMA Biomedicals AG, August, Switzerland), were purchased from Pharmingen. All cells for flow cytometric analysis were pre-incubated with $10 \mu \mathrm{g} / \mathrm{ml}$ of rat anti-mouse $\mathrm{CD} 16 / 32$ antibody $(2.4 \mathrm{G} 2)$ at $4^{\circ} \mathrm{C}$ for $30 \mathrm{~min}$ before being stained with specific antibodies. Cells $\left(1 \times 10^{5}\right)$ were incubated for $60 \mathrm{~min}$ at $4^{\circ} \mathrm{C}$ with either FITC-conjugated antibodies or immunoglobulins (anti-mouse NK1.1; PK136, anti-mouse CD4; RM4-5, anti-mouse CD8; 53.5.8, anti-mouse B220; RA3-6B2, rat IgG1 isotype control; R3-34, rat IgG2a isotype control; R35-95, or mouse IgG2a isotype control; G155-178), PE-conjugated antibodies or immunoglobulins (anti-H-2 ${ }^{b}$; $\mathrm{KH} 95$, anti-H-2K ${ }^{\text {}}$; AF6-88.5, or mouse IgG2a isotype control; G155-178), APC-conjugated antibodies or immunoglobulins (anti-mouse CD3e; 145-2C11, anti-mouse CD11b; M1/70, hamster isotype control; A19-3, or rat IgG2b isotype control; A95-1) in combination with Via Probe ${ }^{\mathrm{TM}}$ (Pharmingen). After washing with PBS, cells were analyzed using a FACScalibur flow cytometer and CellQuest software (Becton-Dickinson Japan, Tokyo, Japan).

Monoclonal antibody purification. For antibody purification, hybridoma cells were cultured in CD hybridoma serum-free culture medium (Gibco) supplemented with L-glutamate (Gibco), and penicillin-streptomycin (Gibco), and the supernatant was concentrated using an Amicon ${ }^{\circledR}$ model 8200 ultraconcentration module and YM-30 membrane (Millipore, Bedford, MA) and then dialyzed in $0.02 \mathrm{M}$ phosphate buffer, $\mathrm{pH}$ 8.0. The supernatant was passed through a Hi-Trap protein $\mathrm{G}$ affinity column (Amersham Biosciences, Piscataway, NJ) and washed with $0.02 \mathrm{M}$ phosphate buffer, $\mathrm{pH}$ 8.0. The antibody was eluted from the column by $0.02 \mathrm{M}$ citrate buffer, $\mathrm{pH}$ 2.0. The eluted fraction was dialyzed into PBS. Antibody concentration was determined by DC protein assay reagents (Bio-Rad, CA).

In vivo depletion of NK cells, T cells, and macrophages. From 3 days before tumor inoculation, $50 \mu \mathrm{l}$ of anti-asialo GM1 rabbit polyclonal antibody (Wako, Osaka, Japan) dissolved in $1.6 \mathrm{ml}$ of PBS/bottle was intraperitoneally administered every 2 days. In terms of the purified monoclonal antibodies, from 5 days before tumor inoculation, $100 \mu 1$ of $500 \mu \mathrm{g} / \mathrm{ml}$ of purified antibody was intraperitoneally administered every
Table I. The concentration of IL-2 in supernatant of B16 sublines.

Cells $5 \times 10^{5}$ cells $/ \mathrm{ml}$ for $24 \mathrm{~h}(\mathrm{ng} / \mathrm{ml})$

\begin{tabular}{lc}
\hline B16/mock & ND \\
B16/IL-2 & $4.85 \pm 0.30$ \\
\hline
\end{tabular}

ND, not detected.

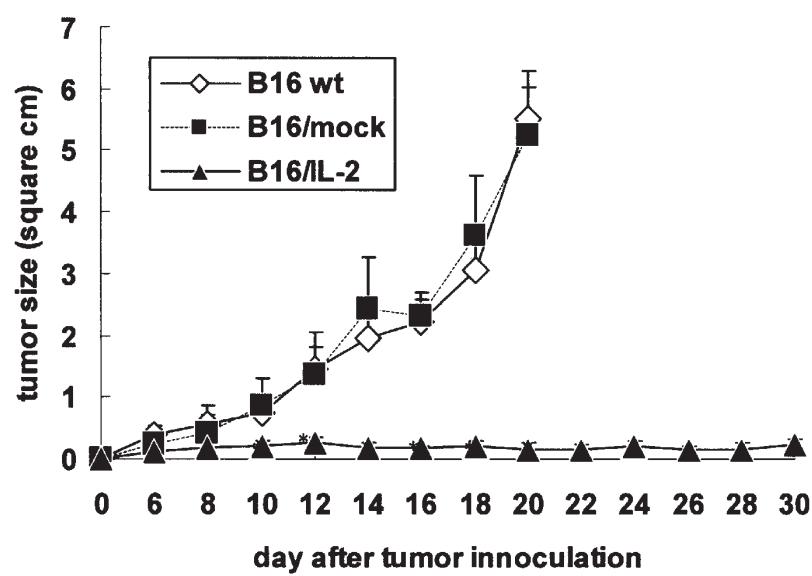

Figure 1. Tumor growth of subcutaneously inoculated B16 tumor cell lines. One million cells of B16/mock, IL-2 producing B16/IL-2 were subcutaneously inoculated to the abdominal flank of C57BL/6 mice. Long and short axes of tumors were measured and tumor sizes were plotted. Each asterisk means there is a statistical significance between 2 groups.

2 days. One hundred microliters of PBS was administered using the same interval, as a control.

Apoptosis analysis. Two hundred and fifty thousand cells of B16 were plated onto a 24-well culture plate with and without recombinant IFN $\gamma$ (Pharmingen). Twenty-four hours after culture, the cells were harvested. After washing with Annexin binding buffer (0.01 M HEPES, 0.14 M NaCl, $2.5 \mathrm{mM} \mathrm{CaCl}$, $\mathrm{pH}$ 7.4), the cells were stained with PE-conjugated Annexin $\mathrm{V}$ (Pharmingen) and Via-probe (Pharmingen) for $15 \mathrm{~min}$ at $4^{\circ} \mathrm{C}$. After washing with Annexin binding buffer, the cells were analyzed by flow cytometry.

Statistical analyses. For statistical analysis, we used the Student's t-test. $\mathrm{P}$ values less than 0.05 were considered significant.

\section{Results}

IL-2 cDNA transduction resulted in reduction of tumor growth in vivo. To observe the effect of IL-2 on tumor growth in vivo, we transduced IL-2 cDNA into B16 and established IL-2 single transfected (B16/IL-2) and vector vehicle alone transfected (B16/mock) lines by a limiting dilution method described in Materials and methods. The cell lines used in this study showed the same growth speed in vitro (data not 

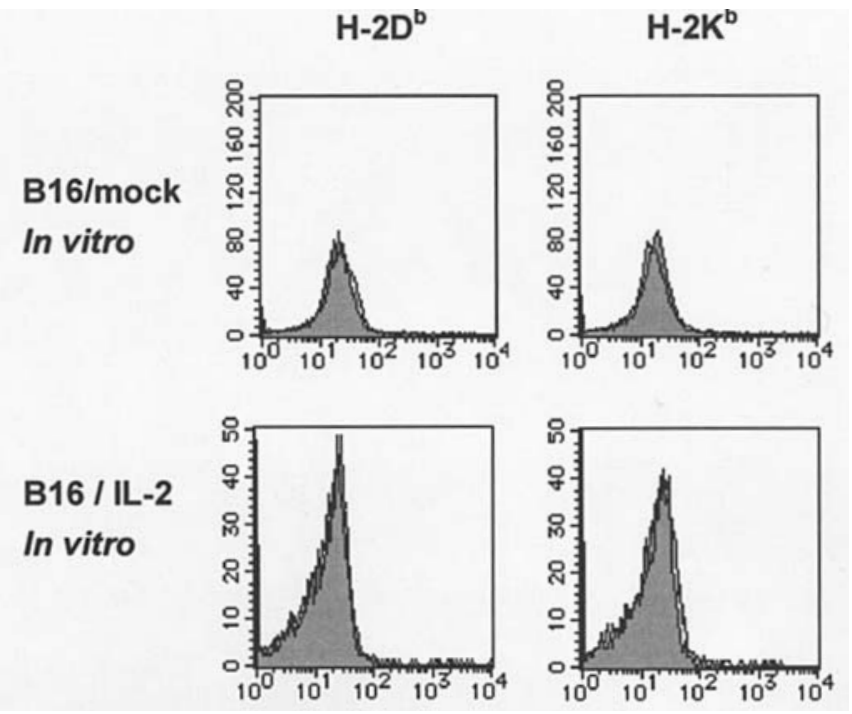

Figure 2. MHC class I expression on B16/mock and B16/IL-2 cells in vitro. Cultured tumor cells were harvested with $0.025 \%$ trypsin and cultured in non-coating Petri dishes to recover cell surface antigen before being subjected to immunofluorescent staining followed by flow cytometric analysis.

shown). The level of IL-2 secretion in the B16/IL-2 cultured supernatant at $5 \times 10^{5}$ cells $/ \mathrm{ml}$ after $24 \mathrm{~h}$ is summarized in Table I. We inoculated B16/IL-2 or B16/mock subcutaneously. B16/IL-2 was not rejected but significantly decreased in size 10 days after tumor inoculation and remained at this size until observation was terminated at 60 days after tumor inoculation (Fig. 1).

In vivo environment made B16 cells express MHC class I molecules. In order to exclude the possibility of IL-2 itself
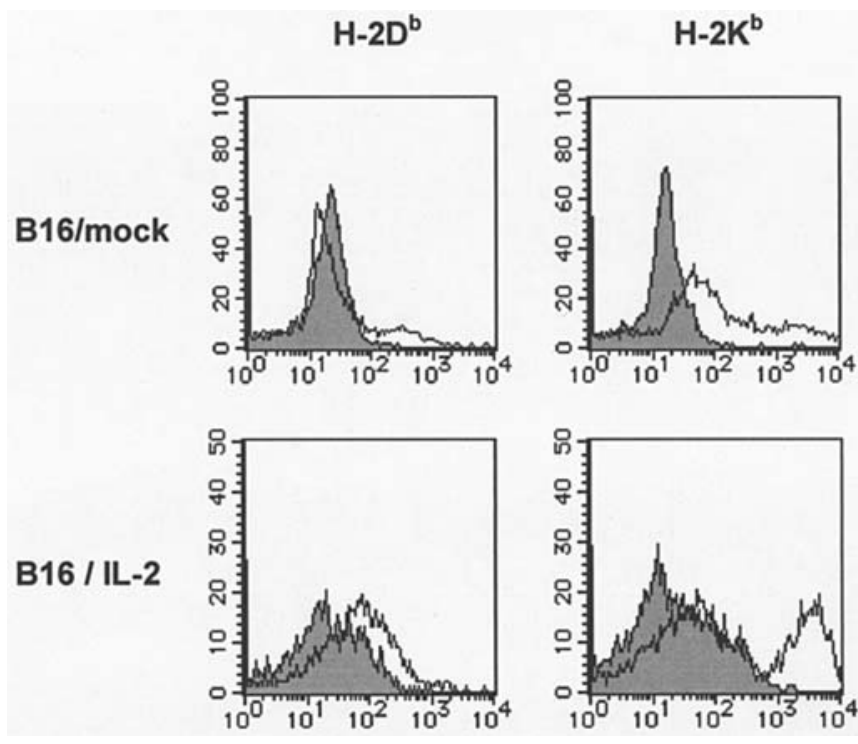

Figure 3. MHC class I staining of in vivo tumor cells. Nine days after inoculation, tumor tissue was harvested and negatively selected total tumor digest was stained with PE-conjugated MHC class I, or control IgG.

causing B16 cells to express MHC class I molecules, we checked the expression of MHC class I molecules for each cell line in vitro. As shown in Fig. 2, the IL-2 secreting line as well as B16/mock did not express MHC class I molecules in vitro, while B16/mock weakly and B16/IL-2 strongly expressed MHC class I molecules in vivo (Fig. 3).

NK cells were indispensable for induction of MHC class I molecules on tumor cells in non-immunotherapy conditions although IL-2 immunotherapy could bypass the role of NK

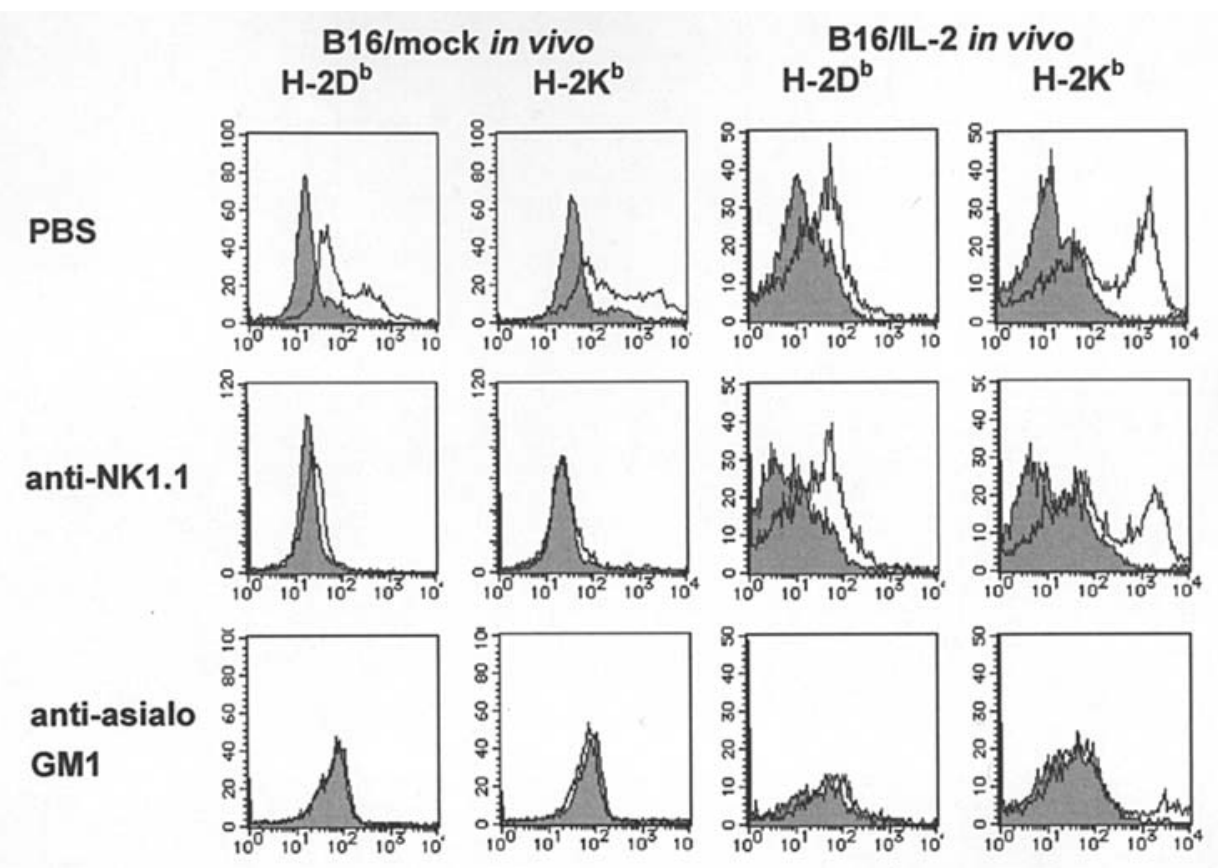

Figure 4. MHC class I staining of in vivo tumor cells after anti-NK1.1 or anti-asialo GM1 treatment. PBS, anti-NK1.1 or anti-asialo GM1 was administered from 3 days before until 6 days after tumor inoculation at 2-day intervals. Nine days after inoculation, tumor tissue was harvested and negatively-selected total tumor digest was stained with PE-conjugated MHC class I or control IgG. 


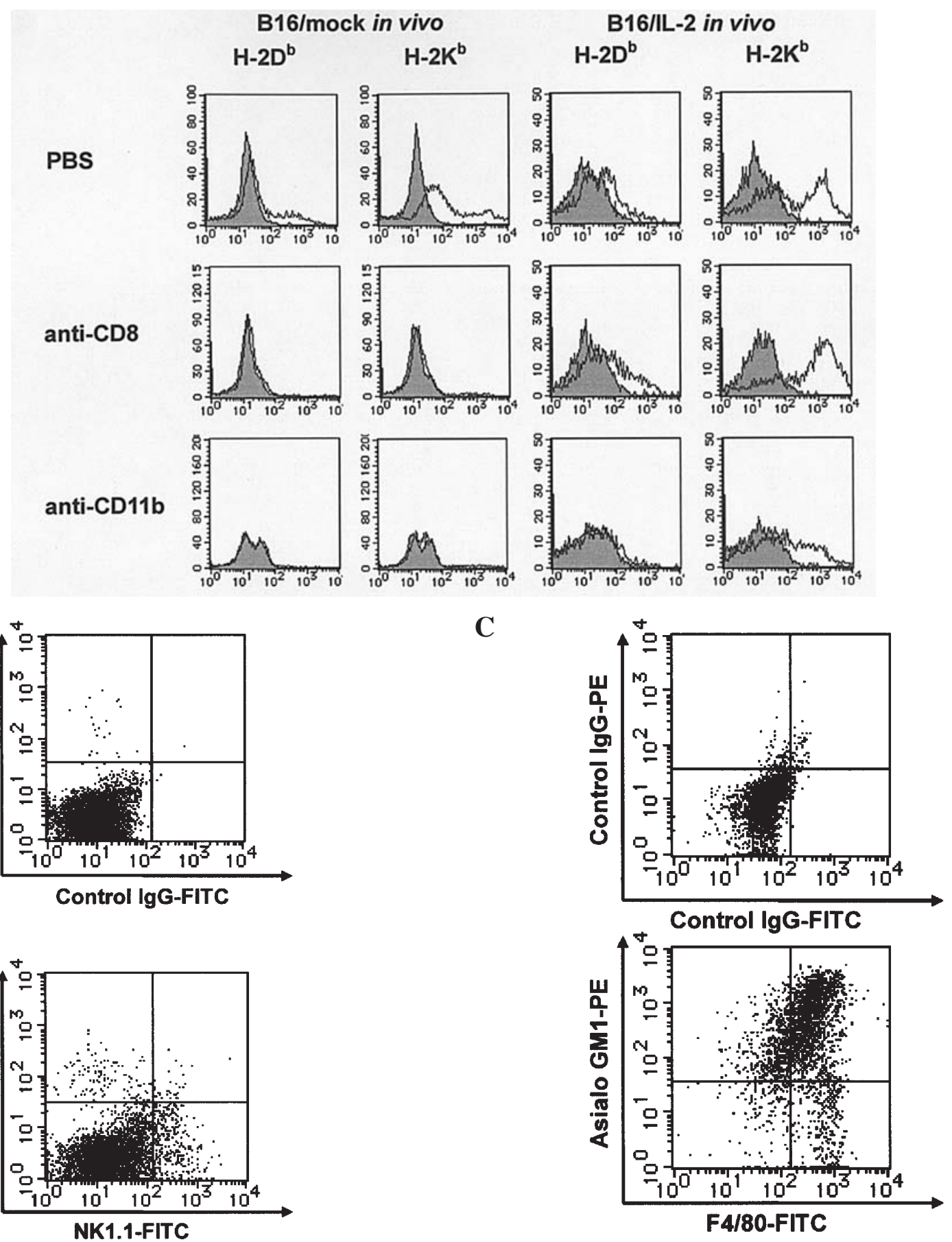

Figure 5. Tumor associated macrophage expressed asialo GM1 antigen and anti-CD11b treatment decreased MHC class I expression of tumor cells in vivo. (A) PBS, CD8 or CD11b antibody was administered from 3 days before until 6 days after tumor inoculation at 2-day intervals. Nine days after tumor inoculation, tumor tissue was harvested and negatively-selected total tumor digest was stained with PE-conjugated MHC class I, or control IgG. (B and C) Nine days after tumor inoculation, CD11b positive cells were separated by magnetic beads positive selection, then stained with the antibody shown in each figure in combination with anti-CD11b and Viaprobe for flow cytometric analysis.

cells for MHC class I induction. In order to clarify the cell responsible for the expression of MHC class I molecules on tumor cells, we depleted NK cells with anti-asialo GM1 antibody or anti-NK1.1 antibody administration. Interestingly, both anti-asialo GM1 and anti-NK1.1 antibody treatment completely abolished MHC class I expression on tumor cells in the B16/mock case. However, not anti-NK1.1 but antiasialo GM1 treatment decreased MHC class I expression on B16/IL-2 tumor cells (Fig. 4).
Macrophage depletion decreased the induction of MHC class I molecules on tumor cells in IL-2 immunotherapy conditions. In order to clarify the cell responsible for the expression of MHC class I molecules on tumor cells in IL-2 immunotherapy conditions, we depleted CD4- (data not shown), CD8-, or CD11b-positive cells. Interestingly, the CD11b depleted condition decreased the expression label of MHC class I molecules on the tumor cells in vivo (Fig. 5A). In addition, CD11b-positive cells contained F4/80-positive macrophages 

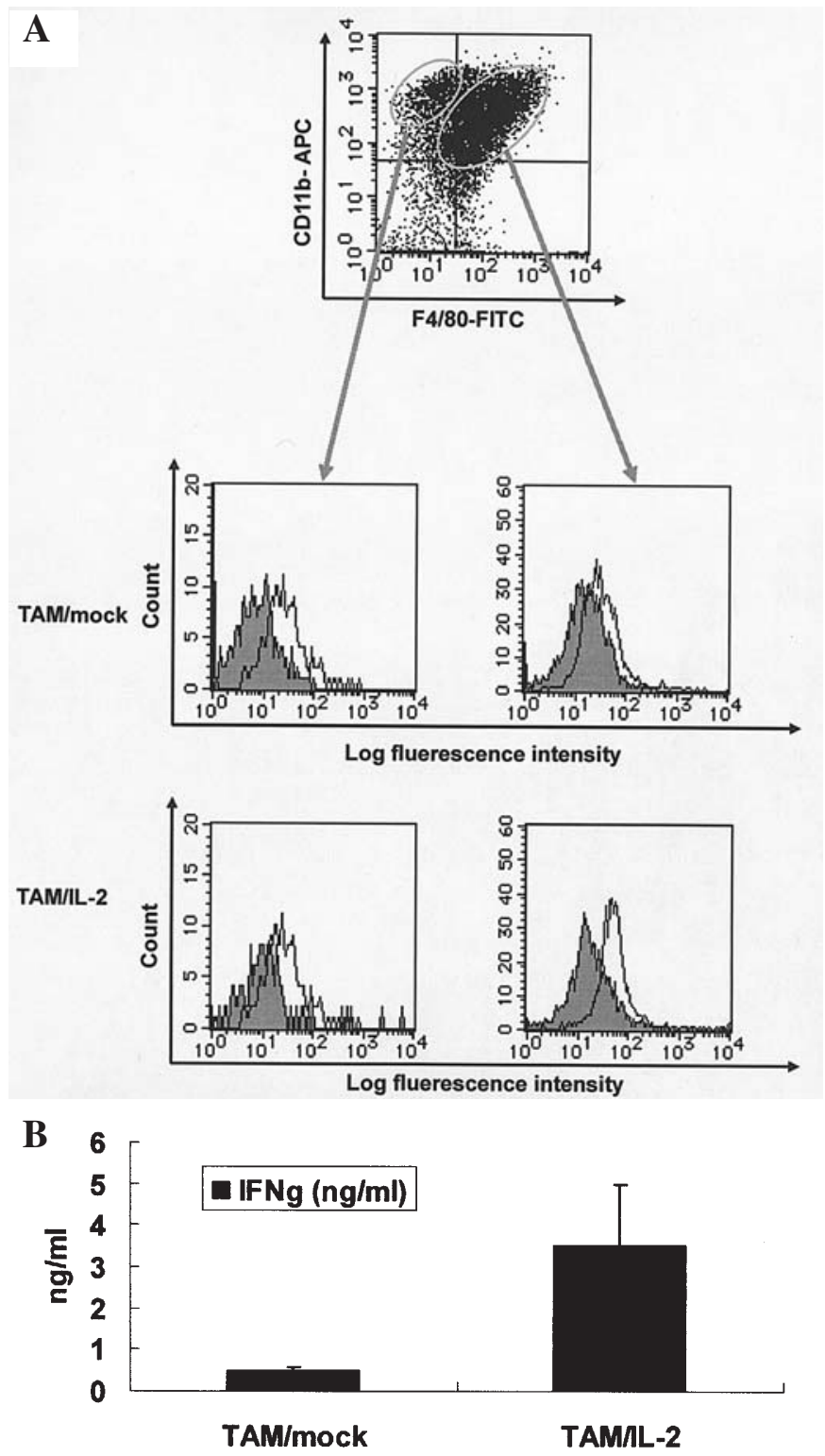

C

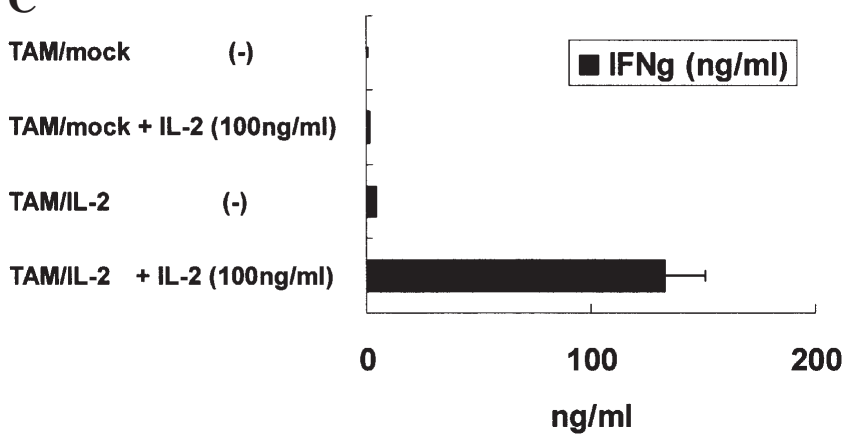

Figure 6. Flow cytometric analysis and ELISA assay for the detection of IFN $\gamma$ in tumor associated macrophage. Nine days after tumor inoculation, CD11b-positive cells were separated by magnetic beads positive selection. (A) Intracellular staining of IFN $\gamma$. (B) The cells were cultured for $48 \mathrm{~h}$ at $3 \times 10^{6}$ cells $/ \mathrm{ml}$ and the supernatants were analyzed. (C) The cells were cultured for $48 \mathrm{~h}$ at $3 \times 10^{6}$ cells $/ \mathrm{ml}$ with or without in vitro administration of IL-2 (100 ng/ml) and the supernatants were analyzed.

and NK1.1-positive NK cells (Fig. 5B). Furthermore, not only NK $1.1^{+}$cells but also macro-phages expressed asialo GM1 antigen on the cell surface (Fig. 5C).
Tumor associated macrophages weakly secreted IFN in normal conditions but strongly secreted IFN $\gamma$ in the IL-2 existing condition in vivo. We observed whether or not TAM could secrete IFN $\gamma$ or not. As shown in Fig. 6A and B, we detected weak and small amounts of IFN $\gamma$ production by intracellular staining of IFN $\gamma$, and ELISA assay for primary culture supernatant in the case of B16/mock recovered TAM. On the other hand, IFN $\gamma$ production and secretion were significantly up-regulated in the case of TAM recovered from B16/IL-2. Interestingly, IL-2 administration during culture of control group-derived TAM weakly up-regulated the IFN $\gamma$ production, while the same treatment for the TAM recovered from B16/IL-2 resulted in strong IFN $\gamma$ production in vitro (Fig. 6C).

IL-2 receptor expression pattern of tumor associated macrophage was different in IL-2 immunotherapy conditions in comparison to normal conditions. We observed which IL-2 receptor (IL-2R) was expressed on TAM. As shown in Fig. 7 (top left panel), the TAM recovered from B16/mock expressed IL-2R $\alpha$ and $\gamma$ chain (CD25 and CD132, respectively). In contrast, the TAM recovered from B16/IL-2 expressed IL-2Rß (CD122) and $\gamma$ chain.

B16 tumor cells became apoptotic with in vitro IFNy treatment. Finally, we checked whether or not B16 cells were sensitive to IFN $\gamma$ treatment. As shown in Fig. 8, IFN $\gamma$ dose-dependently induced B16 tumor cell death in vitro.

\section{Discussion}

In the present study, we focused on the phenomenon related to induction of MHC class I expression on tumor cells in vivo. In the previous study, we showed that IL-2 immunotherapy was effective for increasing NK cell activation and infiltration (13). In that report, we showed that NK cells were important for inducing MHC class I expression on tumor cells in nonimmunotherapy conditions. However, we could not show the reason why IL-2 immunotherapy strongly induced MHC class I expression on tumor cells in comparison to normal conditions (13). NK1.1 antigen was expressed dominantly on NK cells and NKT cells $(15,16)$. Therefore, in order to clarify that NK cells played a critical role in expressing MHC class I molecules on tumor cells, we depleted NK1.1+ cells by anti-NK1.1 antibody in behalf of utilizing anti-asialo GM1 antibody because there was a report that asialo GM1 antigen was expressed not only on NK cells but also on macrophages (17). As shown in Fig. 4, anti-NK1.1 antibody treatment did abolish MHC class I expression on tumor cells in normal conditions as well as anti-asialo GM1 treatment. However, anti-NK1.1 antibody treatment never decreased the MHC class I molecule expression in the IL-2 existent condition although anti-asialo GM1 treatment did decrease MHC class I expression. These findings suggested that the NK cell itself was not the critical player in the induction of MHC class I expression on tumor cells in IL-2 existing conditions in vivo. We further tried to deplete macrophages by anti-CD11b antibody administration because macrophage was the largest $\mathrm{CD}_{11} \mathrm{~b}^{+}$cell population in the tumor environment $(1,14,18)$. As shown in Fig. 5A, anti-CD11b antibody treatment gave 
TAM / mock

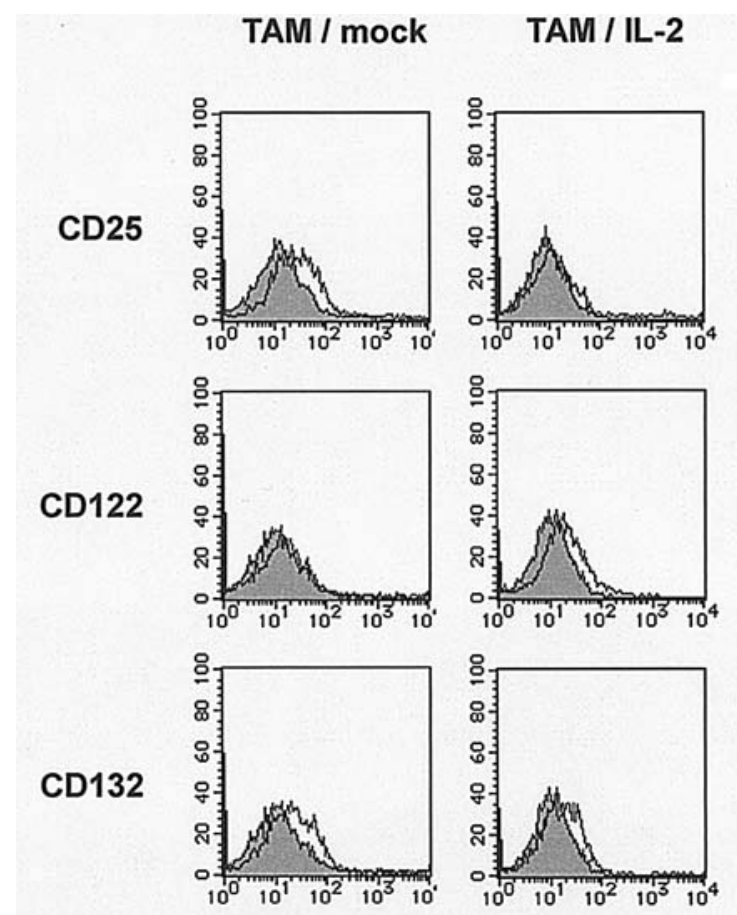

Figure 7. IL-2 receptor expression on tumor associated macrophages. Nine days after tumor inoculation, CD11b-positive cells were separated by magnetic beads positive selection and then flow cytometric analysis was performed to examine IL-2 receptor expression.

rise to a decrease in MHC class I molecules on tumor cells, while neither $\mathrm{NK} 1.1^{+}$nor $\mathrm{CD}^{+}$cell-depletion decreased the expression. These results suggested that macrophages could play a critical role in inducing MHC class I expression in the IL-2 existing condition. Because IFN $\gamma$ is well known for its function to induce MHC class I on tumor cells $(19,20)$, we clarified whether $\mathrm{CD} 11 \mathrm{~b}^{+}$cells produced IFN $\gamma$ in IL-2 existing

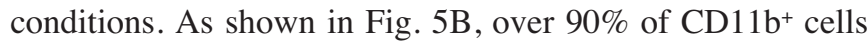
were $\mathrm{F} 4 / 80^{+}$cells, which expressed $\mathrm{F} 4 / 80$ antigen predominantly on mature macrophages (14). Moreover, we confirmed intra-cellular expression of IFN $\gamma$ in $\mathrm{CD} 11 \mathrm{~b}^{+} \mathrm{F} 4 / 80^{+}$ cells recovered from B16/IL-2 (Fig. 6A). Furthermore, both $\mathrm{CD} 11 \mathrm{~b}^{+} \mathrm{F} 4 / 80^{-}$and $\mathrm{CD} 11 \mathrm{~b}^{+} \mathrm{F} 4 / 80^{+}$populations expressed similar levels of IFN $\gamma$ (Fig. 6A). This means that, although $\mathrm{CD} 11 \mathrm{~b}^{+}$population recovered from B16/IL-2 contained $10 \%$ of NK cells, NKT cells and T cells, approximately $90 \%$ of the CD11b-positive cells were an IFN $\gamma$-secreting macrophage population in IL-2 existing conditions (Fig. 5B and 6A). These results suggested that TAM in B16/IL-2 played the main role in induction of MHC class I on tumor cells through production of IFN $\gamma$.

Macrophage activation is classified into 5 groups; innate activation, humoral activation, classical activation, alternative activation, and innate/aquired deactivation (2,21). Classical activation of macrophages was defined as activation by IFN $\gamma$ and stimulation through IFN $\gamma$ resulting in secretion of proinflammatory cytokines (i.e. IL-6, TNF $\alpha$, and IL-1) and nitric oxide (NO) (21). In addition, IFN $\gamma$ up-regulates the IFN $\gamma$ production from peritoneal macrophages (22), which means that IFN $\gamma$-mediated classical activation of macrophages upregulates the IFN $\gamma$ production from macrophages. In the

\section{IFN $\gamma$ treatment}

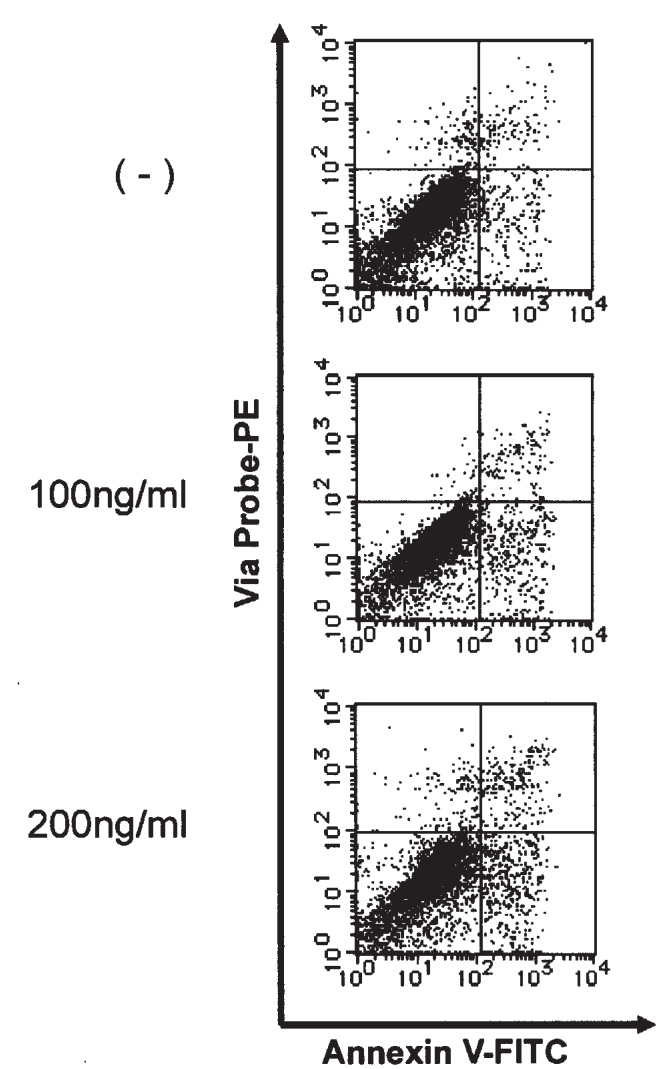

Figure 8. In vitro treatment of IFN $\gamma$ to B16 melanoma cells. The cells were treated with $0,100,200 \mathrm{ng} / \mathrm{ml}$ of recombinant IFN $\gamma$ for $24 \mathrm{~h}$ and then apoptosis analysis was performed.

present study, we showed that the TAM recovered from B16/IL-2 produced IFN $\gamma 6$ times higher in vivo in comparison to the TAM recovered from B16/mock (Fig. 6B). Moreover, in vitro IL-2 treatment strongly induced the IFN $\gamma$ secretion of the TAM recovered from B16/IL-2, although the same treatment slightly induced the IFN $\gamma$ production of the TAM recovered from B16/mock (Fig. 6C). As shown in Fig. 7, the TAM recovered from B16/mock expressed CD25 and CD132 while the TAM recovered from B16/IL-2 expressed CD122 and CD132. CD25 and CD132 compose low affinity IL-2R and CD122 and CD132 compose middle affinity IL-2R (23). In addition, IFN $\gamma$ induced middle affinity IL-2R on macrophages (24). Therefore, the above reports and our data suggest that: i) IL-2 induces production of IFN $\gamma$, ii) the produced IFN $\gamma$ induces composition of middle affinity IL-2R, iii) the composed middle affinity receptor captures high amounts of IL-2 in comparison to the originally employed low affinity receptor, and iv) the captured IL-2 induces further production of IFN $\gamma$ in vivo; i.e. IL-2 and IFN $\gamma$ synergize their effects on the activation process of TAM. Han et al reported that IL-2 enhances IFN $\gamma$-mediated macrophage cytotoxicity to human ovarian carcinoma in vitro (25). Recently, Puddu et al reported that IL-2 induced expression and secretion of IFN $\gamma$ in macrophages (26). These reports support our interpretation. In addition, as shown in Fig. 8, IFN $\gamma$ treatment induced tumor cell death in a dose-dependent manner. This 
means that TAM activated through IL-2 immunotherapy could contribute to B16 cell death induction through its IFN $\gamma$ production if the tumor cells are sensitive to IFN $\gamma$-mediated tumor cell death.

In the present study, we focused on the positive effects of IL-2 immunotherapy. We should also mention the negative effects of IL-2 therapy. For example, it is well known that IL-2 plays an important role in sensitizing $\mathrm{T}$ cells to activation-induced cell death (AICD) by decreasing FLIP [FILCE (Fas-associated death-domain-like IL-1ß-converting enzyme) inhibitory protein] levels during the $S$ phase of the cell cycle (27); the role is mediated through FasL-Fas and $\mathrm{TNF} \alpha-\mathrm{TNF} \alpha$ receptor interactions (28). In addition, in our previous report, we demonstrated that TAM engulfed much CD8 ${ }^{+}$TIL in the IL-2 immunotherapy condition in comparison to control conditions (1). Therefore, we should carefully explore the mechanism by which cytokines influence the immune system and determine how to minimize the negative effects preserving the positive effects of the cytokines before adapting cancer immunotherapy to patients.

In conclusion, in the present study, we clearly demonstrated that it is possible to influence TAM function and induce MHC class I expression on tumor cells in vivo. This is the first report to describe IL-2 mediated activation of TAM playing a main role in the induction of MHC class I expression on tumor cells in vivo.

\section{References}

1. Tamakawa N, Saio M, Suwa T, et al: Interleukin-2 activated microglia engulf tumor infiltrating $\mathrm{T}$ cells in the central nervous system. Int J Mol Med 13: 497-503, 2004.

2. Goerdt S, Politz O, Schledzewski K, et al: Alternative versus classical activation of macrophages. Pathobiology 67: 222-226, 1999.

3. Sica A, Saccani A, Bottazzi B, Polentarutti N, Vecchi A, van Damme J and Mantovani A: Autocrine production of IL-10 mediates defective IL-12 production and NF-kappa B activation in tumor-associated macrophages. J Immunol 164: 762-767, 2000.

4. Verstovsek S, Maccubbin D, Ehrke MJ and Mihich E: Tumoricidal activation of murine resident peritoneal macrophages by interleukin 2 and tumor necrosis factor alpha. Cancer Res 52: 3880-3885, 1992.

5. Kos FJ: Augmentation of recombinant interleukin-2-dependent murine macrophage-mediated tumour cytotoxicity by recombinant tumour necrosis factor-alpha. Immunol Cell Biol 67: 433-436, 1989.

6. Morgan DA, Ruscetti FW and Gallo R: Selective in vitro growth of $\mathrm{T}$ lymphocytes from normal human bone marrows. Science 193: 1007-1008, 1976.

7. Taniguchi T, Matsui H, Fujita T, Takaoka C, Kashima N, Yoshimoto R and Hamuro J: Structure and expression of a cloned cDNA for human interleukin-2. Nature 302: 305-310, 1983.

8. Rosenberg SA and Lotze MT: Cancer immunotherapy using interleukin-2 and interleukin-2-activated lymphocytes. Annu Rev Immunol 4: 681-709, 1986.

9. Whittington $\mathrm{R}$ and Faulds D: Interleukin-2. A review of its pharmacological properties and therapeutic use in patients with cancer. Drugs 46: 446-514, 1993.
10. Rosenberg SA, Yang JC, Topalian SL, et al: Treatment of 283 consecutive patients with metastatic melanoma or renal cell cancer using high-dose bolus interleukin 2. JAMA 271: 907-913, 1994.

11. Janssen RA, Mulder NH, The TH and De Leij L: The immunobiological effects of interleukin-2 in vivo. Cancer Immunol Immunother 39: 207-216, 1994.

12. Hillman GG, Haas GP, Wahl WH and Callewaert DM: Adoptive immunotherapy of cancer: biological response modifiers and cytotoxic cell therapy. Biotherapy 5: 119-129, 1992.

13. Kijima M, Saio M, Oyang GF, et al: Natural killer cells play a role in MHC class I in vivo induction in tumor cells that are MHC negative in vitro. Int J Oncol 26: 679-684, 2005.

14. Saio M, Radoja S, Marino M and Frey AB: Tumor-infiltrating macrophages induce apoptosis in activated CD8(+) T cells by a mechanism requiring cell contact and mediated by both the cell-associated form of TNF and nitric oxide. J Immunol 167: 5583-5593, 2001

15. Vicari AP and Zlotnik A: Mouse NK1.1 ${ }^{+} \mathrm{T}$ cells: a new family of T cells. Immunol Today 17: 71-76, 1996.

16. Yu YY, Kumar V and Bennett M: Murine natural killer cells and marrow graft rejection. Annu Rev Immunol 10: 189-213, 1992.

17. Ecsedy JA, Yohe HC, Bergeron AJ and Seyfried TN: Tumorinfiltrating macrophages influence the glycosphingolipid composition of murine brain tumors. J Lipid Res 39: 2218-2227, 1998.

18. Ando H, Saio M, Ohe N, et al: B7.1 immunogene therapy effectively activates $\mathrm{CD}(4+)$ tumor-infiltrating lymphocytes in the central nervous system in comparison with B7.2 gene therapy. Int J Oncol 20: 807-812, 2002.

19. Fruh K and Yang Y: Antigen presentation by MHC class I and its regulation by interferon gamma. Curr Opin Immunol 11: 76-81, 1999.

20. Zoller M, Strubel A, Hammerling G, Andrighetto G, Raz A and Ben-Ze'ev A: Interferon-gamma treatment of B16 melanoma cells: opposing effects for non-adaptive and adaptive immune defense and its reflection by metastatic spread. Int J Cancer 41: 256-266, 1988.

21. Gordon S: Alternative activation of macrophages. Nat Rev Immunol 3: 23-35, 2003.

22. Di Marzio P, Puddu P, Conti L, Belardelli F and Gessani S: Interferon gamma upregulates its own gene expression in mouse peritoneal macrophages. J Exp Med 179: 1731-1736, 1994.

23. Sugamura K, Asao H, Kondo M, et al: The interleukin-2 receptor gamma chain: its role in the multiple cytokine receptor complexes and $\mathrm{T}$ cell development in XSCID. Annu Rev Immunol 14: 179-205, 1996.

24. Matsui K: Role of interleukin-2 receptor expression on macrophages from Salmonella-infected mice. FEMS Immunol Med Microbiol 24: 97-103, 1999.

25. Han X, Wilbanks GD, Devaja O, Ruperelia V and Raju KS: IL-2 enhances standard IFNgamma/LPS activation of macrophage cytotoxicity to human ovarian carcinoma in vitro: a potential for adoptive cellular immunotherapy. Gynecol Oncol 75: 198-210, 1999.

26. Puddu P, Carollo M, Pietraforte I, et al: IL-2 induces expression and secretion of IFN-gamma in murine peritoneal macrophages. J Leukoc Biol 78: 686-695, 2005.

27. Algeciras-Schimnich A, Griffith TS, Lynch DH and Paya CV: Cell cycle-dependent regulation of FLIP levels and susceptibility to Fas-mediated apoptosis. J Immunol 162: 5205-5211, 1999.

28. Lenardo M, Chan KM, Hornung F, McFarland H, Siegel R, Wang $\mathrm{J}$ and Zheng L: Mature T lymphocyte apoptosis - immune regulation in a dynamic and unpredictable antigenic environment. Annu Rev Immunol 17: 221-253, 1999. 